\title{
CASE REPORT: INTRACRANIAL DISSECTION MISSED BY 1.5T MRI AND CT-A. NEW OPPORTUNITIES WITH 3.0T MRI WITH HIGH RESOLUTION T1-FATSUPPRESSION
}

\author{
A.C. Henriksen ${ }^{1}$, M.N. Folke ${ }^{1}$, H. Christensen ${ }^{1}$, I.B. Havsteen ${ }^{2}$, S. Rosenbaum ${ }^{1}$. \\ ${ }^{1}$ Bispebjerg University Hospital, Department of Stroke, Copenhagen, Denmark. \\ ${ }^{2}$ Bispebjerg University Hospital, Department of Neuroradiology, Copenhagen, \\ Denmark.
}

\section{Background and Aims}

Intracranial dissections are a well-known cause of subarachnoid hemorrhage (SAH) and some dissections only consist of an intramural hematoma, without intravascular bulging. Angiography is therefore of little use in diagnosing these cases. Our case compares different imaging methods.

\section{Method}

A 36 year old woman was admitted with thunderclap headache. Prior to admission she had suffered from two weeks of neck pain.

\section{Results}

Computed tomography angiography (CTA) of neck and brain showed an extracranial vertebral artery dissection in the V2 segment. Due to worsening of headache and new on-set photophobia and nausea she was readmitted two days later at a stroke department. CT revealed a SAH around the brainstem. No changes were seen regarding the extracranial vertebral dissection on CTA. Seven days after onset of thunderclap headache a 1.5T MRI with T1-fatsupression only confirmed previous findings. A 3T MRI with high resolution T1-fatsuppression (HR-T1FS) performed next day showed an intramural hematoma in the intracranial part of the vertebral artery (V4 segment), however, not connected to the extracranial vertebral dissection at V2.

\section{Conclusion}

Retrospectively the extracranial dissection may explain the two weeks of neck pain, while the thunderclap headache was caused by the intracranial dissection with secondary SAH.

Our case indicates that 3T HR-T1FS is more sensitive than regular T1FS at 1.5T and can detect dissections with intramural hematoma which can be missed on CTA.This case underlines the need of further investigations when clinical and imaging findings are contradictory. 